\title{
LA FAMILIA Y SU ESTRUCTURA DE PODER: ESTUDIO DESCRIPTIVO EN EL GRAN SANTIAGO
}

\section{RESUMEN}

Fste estuclio describe algunos aspectos de la estructura de poder en matrimonio y/o parejas provenientes de un estrato socioeconómico medio y bajo del Gran Santiago. Se trabajó con muestra represemtativa de parejas que tuvieran hijos en Escuelas Básicas Fiscales. Los resultados arrojan: i) una fuerte tendencia liacia la estructura igualitaria; ii) una relación entrc el éxito del matrimonio percibido por la mujer y la estructura igualitaria; iii) una relación entre cl poder polarizado en la mujer y el mayor consumo de alcohol del mari. do. Estos clatos muestran que la característion tan mentada en América latina del poder del hombre "machismo" no se da en el medin estudiado.

\section{INTRODUGGION}

En nuestro país, prácticamente, no existen líneas de investigación sociológica ni antropológica de la familia. Este es un descuido serio, dado que la familia es la piedra angular de toda sociedad. Desconocemos las influencias que está sufrienclo la familia y cuáles son sus mecanismos de adajtación. No sabcmos cómo se desarrollará en el futuro.

EI interés de este estudio está en conocer la estructura cle poder que tienen los matrimonios y/o parejas del Gran Santiago, en un nivel medio y bajo; ya que el poder en la toma de decisiones facilita la transmisión de normas y valores que perpetúan una cultura. Por lo tanto, el objetivo es describir la conliguración que presenta la estructura de poder en matrimonios del Gran Santiago y mostrar qué relaciones tiene con otras variables.

Hay muchas investigaciones sobre csta materia en otros paises, pero los investigadores 110 han logrado una conceptualización clara.

Investigaciones realizadas en paises desarrollados han encontrado que existe gran tendencia hacia la estructura igualitaria (LvPRI, 1969). Otras investigaciones han encontrado que la estructura igualitaria está relarionada con la mayor satisfacción matrimonial (Blood y Wolfe, 1960), (Safilios" RothsCHLD, 1967), (CENTERs, RIVEN y RoDRigeEz, 1971).

Estas relaciones encontradas varian dentro del país desarrollado según sea el contexto rel grupo en estuclio, especialmentc tratándose de grupos minoritarios y migratorios. Los mexicanos, considerados una clase inferior, en EE. UU., han mantenido los patrones culturales de dominio del hombre sobre la mu. jer y los hijos. El es quien decide y educa al los hijos (Hawkes y TAYLoR, 1975), y no han asimilado las normas igualitarias que dominan en el pais. Igualmentc, otra investigación que fue realizada en EF. UU. por $M$. L. Richmond (1976), en un grupo de cubanos residentes, encontró que el mayor grado de interacción igualitaria se da cuando los recursos del marido son moderados, cuando la mujer trabaja y aporta dinero al hogar, $y$ 
cuando la pareja está fuertemente expuesta a normas igualitarias.

En cambio, en los paises menos desarrollados nos encontramos con otro tipo de estructura de poder. Aqui existe una tendencia a concentrarse el poder en uno $u$ otro miembro de la pareja, según sea el factor con el cual está asociado. En estos países se ha encontrado que a mayor clominio del marido mayor satisfacción matrimonial encuentra la mujer: (Buric y Zecevic, 1967), (SafiliosRothschild, 1967). Mientras más prestigio tiene el marido mayor poder tiene la mujer (Buric y Zecevic, 1967). (SAFilios-RothsCHILd, 1967). Esto último se da en forma inversa en los países desarrollados. En relación al status socioeconómico existe una asociación tanto en los países desarrollados como menos desarrollados. A menor nivel socioeconómico mayor poder del marido, como lo afirma Gillespie (1971), habiendo encontrado un mayor poder del hombre en los sectores marginales de Estados Unidos.

La orientación teórica utilizada sobre poder es la reconceptualización hecha por Rollins y Bahr (1976), que dice: "el poder estaría diferenciado del control alcanzado, del control propiamente tal, de los recursos y de la autoridad. Ellos postulan que:

a) El poder y el control son interacciones sociales construidas más que atributos individuales de las personas.

b) El poder y el control son construcciones relevantes solamente cuando existe conflicto entre los fines de los compañeros de matrimonio.

c) La autoridad, los recursos y el poder no existen independientes cle las percepciones".

Este trabajo ha utilizado el punio c), ya que se apreciará el poder a través de la perrepción que de él tenga la mujer. Ella percibirá el poder tanto el suyo propio, como el del marido, según sea el control alcanzado por uno u otro en la toma de decisiones.

\section{METODOLOGIA}

El diseño de esta investigación es descriptivo y requiere de una muestra representativa.

El inuestreo se efectuó en forma estratificada y aleatoria de Escuelas Públicas, obteniendo una muestra de 472 matrimonios y/o parejas del Area Metropolitana de Santiago. Este universo permite estudiar las caracteristicas más relevantes de una gran nuasa poblacional urbana de estrato medio y bajo, a través de la mujer.

La técnica utilizada fue uni entrevista estructurada que contenía preguntas abiertas, cerradas y de autoevaluación de la propia entrcvistada. El conteniclo abarcó los aspectos sociofamiliares más importantes de nuestra cultura. La aplicación del instrumento esturo a cargo de 8 entrevistadores debidamente adiestrados. La recolccción de los datos se realizó en 1974.

La estructura de poder se estudió sobre la base de 9 decisiones respecto a:

1. Organización del hogar.

2. Cuidado rle los hijos.

3. Distribución del presupuesto.

4. Distracciones.

5. Vacaciones.

6. Elección de amigos.

7. Proyectos del futuro.

8. Relación con los amigos, y

9. Relación con los parientes.

El vaciamiento de los datos se hizo a través de una escala (le poder de 5 grados, que se presentó a las cntrevistadas, para que contestaran conformc a dicha escala. La gradación de la escala fue la siguientc:

]. Siempre el marido

2. Marido igual que la mujer

3. Ambos en igual medida

4. Mujer más que el marido

5. Sienıpre la mujer

El tratamiento que se hizo a csta escala fue obtener un índice de 3 puntos. La valoración que se dio fue: 
1 punto - 1 y 5 de la escala

2 punto -2 y 4 de la escala

3. punto - 3 de la escala.

El total de puntos que se podia obtener era 27 puntos, significando este puntaje el máximo de igualdad.

La forma de analizar los datos fue:

a) El indice obtenido se dividió en cuartiles para ver la tendencia general del grupo.

b) E1 inclice fue separado por sexo, marido y mujer (siendo 18 puntos el máximo) para determinar en quién se polarizaba el poder. Esta polarización se encontró a partir de los 18 puntos*.

\section{RESULTADOS}

Antes de entrar al análisis de los datos, se presentará el nivel socioeconómico encontrado en las parejas estudiadas.

Este nivel se obtuvo a través de un índice que consicleró sólo las variables atingentes al marido: educación, actividad, previsión social, vivienda (propiedad, tipo calidad, agua, disposición de excretas y de basuras)

La muestra se distribuyó como sigue:

\begin{tabular}{lr} 
Nivel de Vida: & $\%$ \\
\hline Bajo & 4.1 \\
Bajo Alto & 34.1 \\
Medio & 52.4 \\
Medio Alto & 9.4
\end{tabular}

A. Estructira de PoDER.

Los resultados muestran que más de la mitad de las parejas $(57.7 \%$ ) poseen una estructura igualitaria y un $36,2 \%$ tiende a esa igualdad (Tabla 1).

Tabla 1

ESTRUCTURA DE PODER

\begin{tabular}{lrr} 
Calificación & Fr & $\%$ \\
\hline Polarizada & 29 & 6.1 \\
Tendencia a la igualdad & 171 & 36.2 \\
Igualdad & 272 & 57.7 \\
\hline TOTAL & 472 & 100.0 \\
\hline
\end{tabular}

Las variables que se presentan fuertemente relacionadas con esta estructura es: la apreciación que tiene la mujer de su propio matrimonio como un éxito: a mayor igualdad en la toma de decisiones, mayor apreciacion de su matrimonio como éxito (Tabla 2); la menor

Tabla 2

APRECIACION DEL PROPIO MATRIMONIO Y ESTRUCTURA DE PODER

\begin{tabular}{|c|c|c|c|c|c|c|c|c|}
\hline \multirow{4}{*}{$\begin{array}{l}\text { Apreciación } \\
\text { matrimonio } \\
\text { como: }\end{array}$} & \multicolumn{6}{|c|}{ ESTRUCTURA DE PODER } & \multirow{2}{*}{\multicolumn{2}{|c|}{$T \circ t a l$}} \\
\hline & & & & & & & & \\
\hline & \multicolumn{2}{|c|}{ Polarizada } & \multicolumn{2}{|c|}{ Igualdad } & \multicolumn{2}{|c|}{ Igualdad } & \multirow[t]{2}{*}{$F$} & \multirow[t]{2}{*}{$\%$} \\
\hline & $F_{T}$ & $\%$ & $F r^{\prime}$ & $\%$ & $F r$ & $\%$ & & \\
\hline Todo un éxito & 4 & 3.5 & 29 & 23.6 & 89 & 72.9 & 122 & 100.0 \\
\hline Un éxilo & 11 & 5.5 & 72 & 34.5 & 126 & 60.0 & 209 & 100.0 \\
\hline Más o menos un éxito & 7 & 6.8 & 51 & 49.6 & 45 & 43.6 & 103 & 100.0 \\
\hline I'n fracaso & 4 & 16.0 & 12 & 48.0 & 9 & 36.0 & 25 & 100.0 \\
\hline Total fracaso & 2 & 22.2 & 4 & 44.5 & 3 & 33.3 & 9 & 100.0 \\
\hline TO'TAL & 28 & 5.0 & 168 & 35.9 & 272 & 59.1 & 468. & 100.0 \\
\hline
\end{tabular}

$\mathrm{x}_{0}^{2}=32,8998 \quad \mathrm{p}<0.001 \quad$ d.f. 4

* La diferencia de $10 \%$ es significativa por tener un $\mathbf{N}$ mavor de 400. 
frecuencia de discusiones: a mayor igualdad en Ia toma dé decisiones, menor frecuencia de discusiones entre la pareja; la elección de los amigos y la relación con ellos (Tabla 3) se

Tabla 3

\section{ESTRUCTURA DE PODER EN LA ELECCION DE AMIGOS}

\begin{tabular}{|c|c|c|}
\hline Estructura de Pader & $F r$ & \% \\
\hline Polarizada marido & 139 & 34.0 \\
\hline Igualdad & 231 & 56.0 \\
\hline Polarizada mujer & 40 & 10.0 \\
\hline Sin información o & & \\
\hline no tiene amigos.. & 62 & $\cdots, \cdots$ \\
\hline TOIAL & 472 & 100.0 \\
\hline
\end{tabular}

presentan como variables relacionadas con la estructura de porler igualitaria. Aclemás, se encuentra una ielación entre la organización de! hogar y la educación de los hijos con la estructura igualitaria (Tabla 4 ).

\section{B. Poder polarizado.}

Este poder queda manifestado en el control que posee un miembro de la pareja sobre otro. Los resultados muestran que existe una relación entre el dominio de la mujer y el fracaso matrimonial: a mayor poder de la mujer (en los casos de polarización), mayor apreciación de su matrimonio como un fracaso; la relación entre cl consumo de alcohol del marido y el dominio de la mujer es marcado: a mayor poder de la mujer, mayor frecuencia de consumo de alcohol por parte del marido (Tabla 5).

No se presentaron relaciones entre la es. tructura de poder y nivel sociocconómico, actividad del marido y/o mujer, nivel de escolaridad del marido y/o mujer, edad del marido y de la mujer. Se hace notar que estas variables aparecen relacionadas con la estructura de poder en los países desarrollados y menos desarrollados.

Tabla 4

URGANIZACION DE HOGAR Y EDLCACION DE LOS HIJOS SEGLN LA ESTRLCTLRA DE PODER

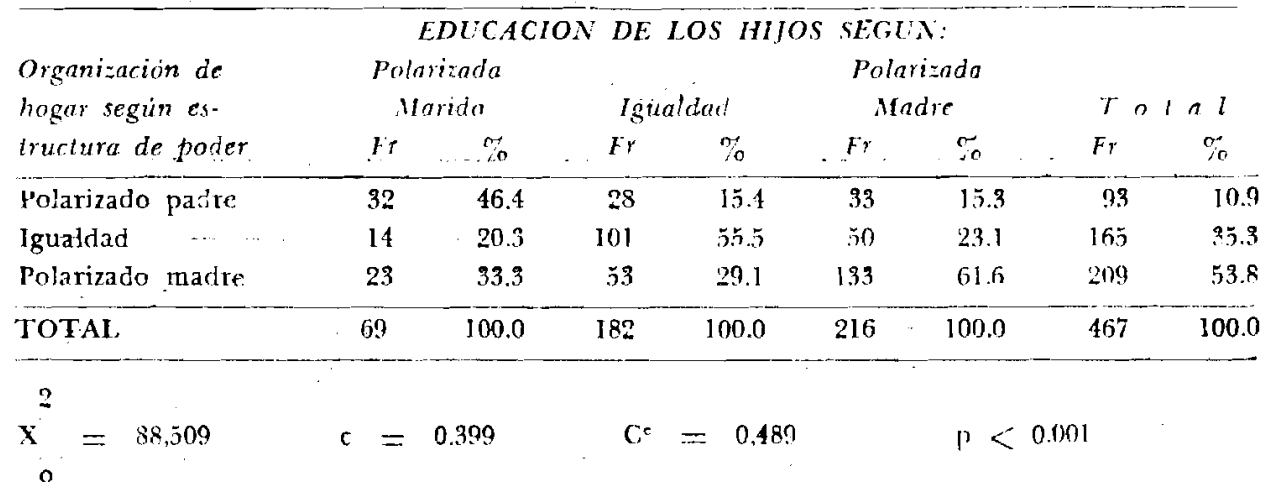

\section{DISCUSION}

Los resultados muestran que la estructura "machista" no se da en el nive] socioeconónini. co medio y bajo de la muestra estudiada. Esto refleja que existe una tendencia marcada hacia la estructura igualitaria. Se dan rasgos de paises clesarrollados y otros no. La pregunti que cabe hacerse es ¿por qué se presenta asi? Según A. Mattelart y M. Mattclart (1968) en su estudio sobrc la condición de la mujer chilena, existiría una idea de igualdad entre el hombre y la mujer a torlo nivel de la gama social incluyendo la pob'ación rural. Esta idea involucraría una igualdad de derechos y por lo tanto de responsabilidades. 
FRECLENCIA DE CONSUMO DE ALCOHOL Y ESTRLCTURA DE PODER POL.ARIKIDA

\begin{tabular}{|c|c|c|c|c|c|c|}
\hline \multirow{2}{*}{$\begin{array}{l}\text { Frecuencia } \\
\text { consumo } \\
\text { alcohol }\end{array}$} & \multicolumn{2}{|c|}{$\begin{array}{l}\text { Poder polarizado } \\
\text { marido }\end{array}$} & \multicolumn{2}{|c|}{$\begin{array}{c}\text { Poder polarizado } \\
\text { mujer }\end{array}$} & \multicolumn{2}{|c|}{$T \circ t a l$} \\
\hline & $F r$ & $\%$ & $F$ & $\%$ & $F r$ & $\sigma_{o}^{\prime}$ \\
\hline Nunca y rara vez & 19 & 33.3 & $j$ & 15.6 & 24 & 26.9 \\
\hline \multicolumn{7}{|l|}{ Varias veces al diay } \\
\hline varios seguidos & 2 & 3.5 & 13 & 40.6 & 15 & 16.8 \\
\hline Fines de semanas y fiestas & 26 & 45.6 & 11 & 34.3 & 37 & 41.5 \\
\hline Todos los días en comidas & 10 & 17.6 & 3 & 9.5 & 13 & 14.8 \\
\hline TOTAL & 57 & 100.0 & 32 & 100.0 & 89 & 100.0 \\
\hline \multicolumn{7}{|l|}{2} \\
\hline$x_{o}=20.6940$ & $\mathbf{p}$ & & & 3 & . & \\
\hline
\end{tabular}

Además se hace el alcance que: 1) Nuestra cultura es permeable y abierta a la influencia externa; 2) La mujer chilena siempre ha participado en la vida política y social del país desde la época de los españoles (tanto mujeres indígenas como criollas) hasta nuestros días; 3) La Tv ha comenzado sólo lace 22 años (1954) y extendiéndose en el nivel socioeconómico alto hace 10 años, y a un nivel masi. vo, sólo hace 5 años. Por lo tanto, su influencia en estas parejas no puede ser considerada.

Entonces, cabe preguntarse: ¿esta estructura igualitaria responde a "progreso", como sucede en los países desarrollados, o es un rasgo cultural del medio urbano? Esta interrogante podría ser respondida a través de estudios antropológicos que busquen comunidades rurales y las comparen con comunidades urbanas de semejante nivel cultural.

\section{B I B L. I O G R A F I A}

BELL, R. Marriage and Family Interaction. Homewood. 111. Dorsey. 1969

Bloov, E. and Wolfe, D. Husbands and Wives: The dynamics of married Living Glencoe, nu: Free Press, 1960.

Burr, W. R. Theory construction and Sociology of the Family. John Wiley and Sons, New York, 1973.

Btiric, O. and /ECric, A. Family authority, marital satisfaction and the Social Network in Yugoslavia. Journal of Marriage and the Family 29 (May): $325-336,196$ T

Centers, R.; Raven, B. H. and Rodriguez, A. Conjugal power structure: a re-examination. American Sociological Review 36 (April): 245.253, 1971.

GillespIE, D. Who has the power? The marital strug. gle. Journal of Marriage and the Family. 33 (August) : 445-452, 1971 .

Hawkes, G. R. and TAYLOR, M. Power structure in Mexican and Mexican/American farm labor Families. Journal of the Marriage and the Family 37 (November): 807.811, 1975.

Kolb, T.; Stralss, M. and Mlrray, A. Marital Power and Marital Happiness in relation to problem solving ability. Journal of the Marriage and the Family, 86 (November): 756.766, 1974.

LuPRI, E. Contemporary Authority patterns in the West German family; a study in cross-national validation. Journal of the Marriage and the Family, 31 (February): 134-144, 1969.

Mattelart, A. y Mattelart, M. La mujer chilena el una nucva sociedad. Ed. del Pacífico S.A., Santiago, 1968 . 
Rodman, H. Marital Power in France, Greece, Yugoslavia and the United States: A cross National discussion. Journal of the Marriage and the Family, 29 (May): 320-324, 1967.

ROCERs, M. Instrumental and Infra-Resources: The bases of power. American Journal of Sociology, 79 (May) : 1418-1439, 1974.

Rollins, B. C. and BaliR, S. J. A theory of power relationships in marriage. Journal of the Marriage and the Family, 98 (November): 619-627, 1976.

Richmond, Marie L. Beyond Resource Theory: Another Look at factors: Enablig women to affect interaction. Journal of the Marriage and the $F_{2}$ mily 38 (May) : 257-266, 1976.
Ross, AILeEn D. The substructure of power and autho. rity in marriage the family: A comparation of contemporary problems. Ed. Meyer Barash and A. Scowby. N. York, 1970.

Saficios-Rothschild, C. A comparaison of power struc. ture and marital satisfaction in urban Greak and French families. Journal of the Marriage and the Family, 29 (May): 345.352, 1967.

Safilios-Rothschil.d. C. A macro and micro-examination of Family Power and love; An Exchange Model. Journal of the Marriage and the Family, 38 (May) : 855-362, 1976.

Wolfe, D. Power and authority in the family selected studies in marriage and the family. Holt, Ri. nehart and Winston. N. York, 1962. 